\title{
Network Management Information for Light-Path Assessment: Trade-Off between Performance and Complexity
}

\author{
Guanglei Liu \\ Department of Electrical and Computer \\ Engineering \\ Georgia Institute of Technology \\ Atlanta, GA 30332-0250
}

\author{
Chuanyi Ji \\ Department of Electrical and Computer \\ Engineering \\ Georgia Institute of Technology \\ Atlanta, GA 30332-0250
}

\author{
Vincent Chan \\ Laboratory of Information \\ and Decision Systems \\ Massachusetts Institute of Technology \\ Cambridge, MA 02139
}

\begin{abstract}
We investigate network management information for light-path assessment to dynamically set up end-to-end lightpaths across administrative domains. Our focus is on invetigating what performance can be possibly achieved given partial management information, and whether a small loss in performance can trade off with a large saving in management information.

The partial information we consider includes aggregated characterization of subnetworks, and local states from wavelength converters. We cast the light-path assessment as a decision problem, and define the performance as the probability of an erroneous decision. We apply the decision theory to show that the optimal performance using the partial information is the Bayes probability of error. We derive an upper bound of the Bayes error in terms of the blocking probability. We evaluate the upper bound using both independent and dependent models of wavelength usage. Our study shows that there exits a "threshold effect": The Bayes error decreases exponentially to 0 with respect to the load when the load is either below or above a threshold value; and is non-negligible when the load is in a small duration around the threshold. This suggests that a small percentage of error decisions can trade off with a large saving in management information.
\end{abstract}

Index terms-- Partial management information, lightpath assessment, decision theory, Bayes rule, blocking probability.

\section{INTRODUCTION}

Dynamically assessing the quality of light-paths is important to many applications in wavelength-routed optical networks such as on-demand light-path provisioning, protection and restoration. As the light-path quality is a complex measure [1], this work considers a simple quality, which is the wavelength availability on a candidate light-path. The assessment then becomes determining availability of wavelengths for supporting an end-to-end call based on given management information.

Complete or partial network management information can be used to assess the wavelength availability on a light-path. Complete information corresponds to the detailed states of wavelength usage, i.e. "which wavelengths are used at which links of a network", when there is no wavelengths converters in the network. Wavelength converters can reduce state information due to their ability to relax the wavelength continuity constraint. However, it is expected that wavelength converters remain expensive and are thus used mostly on the boundaries of sub-networks [2]. Therefore, generally complete state information involves the detailed wavelength occupancy within a subnet. Partial information includes aggregated load and topology information at each subnet, and local states, e.g., the total number of wavelengths used at wavelength converters.

Providing state information is a basic functionality of network management. Traditional network management systems intend to obtain as complete state information as possible [3]. But future IP-WDM networks may have hundreds of links with each link supporting hundreds of wavelengths [4]. This would result in a huge amount of state information for networks without wavelength converters. For instance, let $H$ be the number of links within each subnet, $F$ be the number of wavelengths supported per link at each subnet, and $L$ be the number of subnets. The total amount of information about wavelength usage is in the order of FHL. When $F=200, H=250$ and $L=10$, the number of states is about half a million. Storing, and updating even a fraction of such a large number of states may result in an undesirably large amount of management traffic. Therefore, it may be prohibitive to manage a large network using complete state information.

Using partial management information is also a requirement of multi-vendor services. A light-path may trespass multiple administrative domains (sub-networks) run by different service providers. A service provider may prefer to exchange only minimal information to other managed domains rather than share complete state information of its own. In fact, it has been the experience today in the Internet that network managers of different administrative domains are extremely reluctant to and rarely share detailed network state information of their subnets with others. Therefore, interdomain subnets are like network clouds to a service provider (see Figure 1 for illustration) [5]. Light-path assessment may have to use partial information on network clouds since it is infeasible to obtain complete management information across domain boundaries.

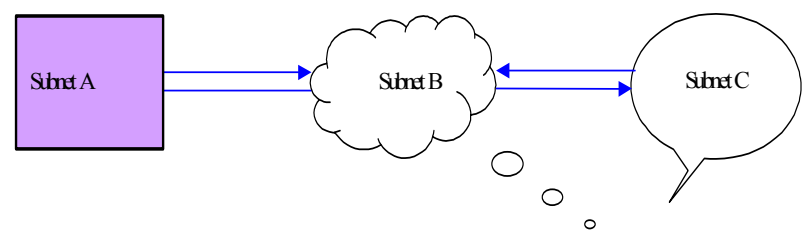

Figure 1: Network Clouds 
Therefore, a fundamental issue is what performance can possibly be achieved given the partial information. More specifically, the related issues are:

(1) What is the best performance of light-path assessments with partial information?

(2) What is the trade-off between the performance and the complexity (i.e., the amount) of network management information?

Little has been studied on how to measure the performance based on the amount of management information ([6] shows a good example). The performance should be defined to evaluate the best performance based on given partial information. Such a best performance would tell the sufficiency or insufficiency of the partial information. Intuitively, when complete information is available, the performance would be the best. When the network management information is reduced, the performance would degrade accordingly. Minimal state information is certainly desirable to keep network management simple. But the simplicity would not be meaningful if the corresponding performance is poor. Therefore, the optimal performance would allow the evaluation of the trade-off between performance and the complexity.

We pose the problem of light-path assessment as a decision problem, and define the performance as the probability of an erroneous assessment. An error occurs when an assessment decision differs from the ground truth (in terms of availability of wavelengths on a given path). The value of the error probability measures a deviation from the optimal performance (with zero error) when the complete information is available, and thus quantifies the sufficiency/insufficiency of the partial management information.

We consider a simple network (bus) topology in this work to study the performance of partial management information. The network bears the same spirit as that in Figure 1. Wavelength converters are only located at the boundaries of, but not within, subnets.

The partial information we consider includes (a) aggregated information on network load (and topology) within subnets, and (b) local state information at wavelength converters. The aggregated information serves as model parameters of wavelength usage, and the local information corresponds to random states or observations obtained locally at domain boundaries. The complexity of management infomaton is evaluated using the number of bits needed to specify the partial information. For a bus topology with $F$ available wavelengths at each subnet and $L$ subnets, the complexity of partial information is $O(\operatorname{Llog} F)$. This is much smaller than $O(F H L)$ for complete state information, especially when the number of wavelengths $F$ is large. The performancecomplexity trade-off is whether a small probability of error can be obtained for most of the network-load conditions when only partial information is used.

To investigate the performance-complexity trade-off, we consider the Bayes decision rule, which results in the Bayes probability of error, the best performance achievable given the partial information. We show that the Bayes error is bounded by $\min \left\{P_{b}, 1-P_{b}\right\}$, where $P_{b}$ is the blocking probability of a light path. This links our performance measure with a metric commonly-used for WDM networks [7] [8] [9]. The (Bayes) probability of error can then be investigated using different models for blocking probability. We first adopt an independent model corresponding to intra-domain calls. We then extend the independent model to a dependent model to include inter-domain calls. For both cases, we show that an important characteristic of the performance-complexity tradeoff is a "threshold effect"'. That is, there exists a threshold for the load. When the load is close to the threshold value, the blocking probability makes a sharp transition from 0 to 1 for $F$ to be sufficiently large. The probability of error decreases accordingly, in fact exponentially to zero with respect to $F$ for most of the load conditions. This suggests that a small loss in performance may be traded off with a large saving in network management information.

The paper is organized as follows. Section II summarizes the prior work. Section III provides problem formulation. Section IV presents Bayes decision theory, and an upper bound of the best performance (the Bayes error) that can be achieved given the partial information. Sections V and VI investigate the best performance using independent model and the dependent model respectively. Section VII concludes the paper.

\section{Related Work}

Various schemes have been proposed for managing IPWDM networks based on different amount of management information. One extreme case is to use complete state information to establish connections [10]. This approach, as discussed earlier, is not feasible for dynamically setting up connections or flows for large networks. Another extreme case is to manage sub-networks as separate entities [11]. But the corresponding performance (i.e., the correctness of an assessment) can be poor due to lack of information. An intermediate approach is proposed to use partial informationexchange among network clouds (subnets) [12]. The idea of using partial information is also investigated in other related applications such as network survivability [13] [14], path protection [15], and path assessment [16]. These works have a different focus, which is mostly on developing approaches to manage networks using partial information. They motivate this work to investigate the performance-complexity tradeoff of management information.

Probing methods have been proposed to obtain information from network clouds [17]. These methods, however, are intrusive, and may be difficult to use for inter-domains due to security reasons. Therefore, management schemes that require minimal probing are desirable.

Wavelength converters (optical or electronic) have been considered as network elements in designing WDM networks to improve wavelength utilization [18]. Sparsely-allocated wavelength converters are found to be sufficient to achieve a desired utilization gain sometimes [19]. The use of 
wavelength converters has also been conjectured to result in simplified network management systems due to their ability to reduce the state information [18]. This motivates us to consider a natural network architecture where wavelength converters are located at the boundaries of subnets (administrative domains).

Prior investigations in other related areas are also beneficial to this research. In particular, inaccurate or aggregated information has been investigated in the context of QoS routing for IP network [6]. Commonly used aggregated information is topology aggregation [20] that can be regarded as a summarized characterization of a subnet. Local information is considered in [21] for QoS routing in IP networks. However, the main focus of aforementioned work is on managing existing (IP) rather than IP-WDM networks.

Therefore, the tradeoff between performance and the complexity of management information has not been investigated quantitatively. In our prior work [22], we formulated the problem of network management information for light-path assessment based on an independent model of wavelength usage. This work extends the prior work to investigate the performance and complexity tradeoff by considering both independent local calls and dependent interdomain calls.

\section{Problem Formulation}

\section{A. Network Architecture}

We consider assessing wavelength availability for an endto-end call request from source $S$ to destination $D$ as shown in Figure 2. Wavelength converters are located at the boundaries of one-dimensional subnets and there are $L$ subnets on a given path. Each subnet has $H$ hops and each link supports $F$ wavelengths.

Such network architecture, although simple, bears the same spirit as in Figure 1. For simplicity, we assume the subnets are identical, although our approach and analysis apply to subnetworks with different parameters $(F, H, L)$.

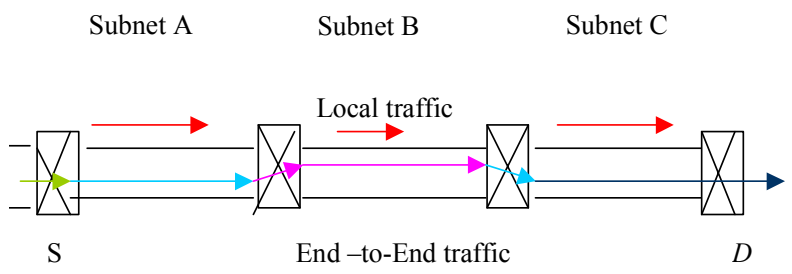

Figure 2: Network Architecture

\section{B. Partial Management Information}

The partial information we consider consists of aggregated and local states. The aggregated information characterizes the average behavior of each network cloud so that detailed network states within each subnet do not need to be maintained. To be more specific, the aggregated information is denoted as $A=\left(A_{1}, A_{2}, \ldots, A_{L}\right)$, where $A_{i}$ is the aggregated information on subnet $i$ and $A_{i}=\left(F_{i}, H_{i}, \rho_{i}, \pi_{i}\right) . F_{i}$ and $H_{i}$ represent the number of wavelengths per link and the number of hops at subnet $i$. $\rho_{i}$ is the probability that a wavelength is used on a link, which is the load information aggregated over all detailed states about wavelength usage within a subnet. $\pi_{i}$ can be other parameters related to wavelength usage. For simplicity of analysis, we assume that each subnet has the same aggregated information. Then $A$ becomes $A=(F, H, L, \rho, \pi)$.

In practice, the aggregated information can be estimated through measurements, which may deviate from true parameters, and thus introduce additional information loss. For simplicity, we regard aggregated parameters to be accurate. These parameters may also change with time but at a much larger time scale than the connection dynamics, and could thus be regarded as nearly static.

The local information corresponds to the number of wavelengths used at the first hop of each subnet, which is readily available at the wavelength converters. Specifically, the local information corresponding to observations (states) at the wavelength converters is given as $X=\left(N_{1}, N_{2}, \ldots, N_{L}\right)$, where $N_{i}$ is the number of wavelengths used at the $i$ th wavelength converters. Such local information is changing with set-up and tear-down of connections, and can thus be considered as random variables.

The local information is informative due to the wavelength continuity constraint within a subnet. For instance, if nearly all wavelengths are used at the first hop of a subnet, we can infer that the load is high and there may not be any wavelength available within the subnet to support an additional end-end call. Likewise, the aggregated information is informative since it characterizes the average load in a subnet. But the aggregated and local information is incomplete in determining network states, resulting in possibly erroneous wavelength assessments.

\section{Decision Problem and Performance}

We pose the light-path assessment as a decision problem. A decision variable $\omega$ is defined as follows: $\omega=1$ if there is one end-to-end wavelength continuous path across subnets for the connection request; and $\omega=0$ otherwise. The problem of light-path assessment is to decide on $\omega$ given the partial information. Then the performance of light-path assessment can be defined as the probability of erroneous decisions.

Definition 1. The probability of error $P_{e}$ is defined as the probability that the assessment decision is different from the ground truth (in terms of availability of wavelengths on a given path). 
Let $D$ be the decision region on the management information $X$ for $\omega=1$; and $\bar{D}$ be the decision region for $\omega=0$. That is, if the observation $X$ falls in $D(\bar{D})$, the decision should be $\omega=1(\omega=0)$. We then have the probability of error

$$
P_{e}=P_{r}(X \in D, \omega=0)+P_{r}(X \in \bar{D}, \omega=1) .
$$

$P_{e}$ characterizes the average performance given the partial information. The validity of such a performance measure can be understood through Figure 3. When the complete information is available, no error is made in assessing wavelength availability, and the performance is the best (i.e., zero error). When no information is available, decisions can only be made based on random guessing, and the performance is the worst (i.e., $50 \%$ error). The value of $P_{e}$ thus measures a deviation from the optimal performance (zero error) when the complete information is available, and thus quantifies the sufficiency/insufficiency of the management information available.

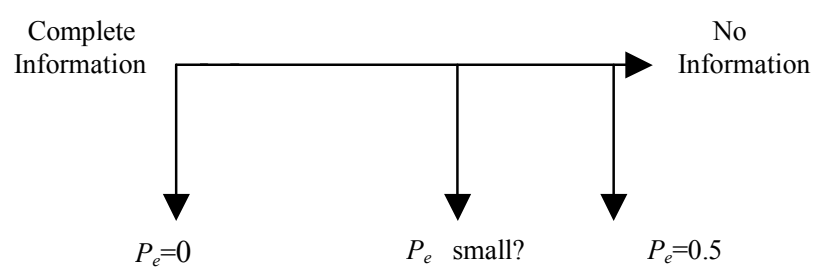

Figure 3: Performance vs. amount of management information

A question is whether it is possible to use partial management information at the cost of a small number of incorrect decisions.

\section{Complexity of Management Information}

We formally define the complexity as follows.

Definition 2: The complexity of management information $Q$ is defined as the total number of bits needed to specify the information.

Using such a measure, we can quantify the complexity of complete and partial information. Consider the network shown in Figure 2. The detail states within each subnet are "which wavelengths are used at which link". The total number of (binary) states is $F H$ for each subnet, and $F H L$ for $L$ subnets. Therefore, the complexity of complete information is

$$
Q_{\text {complete }}=F H L \text {. }
$$

Such complexity is in the order of $F$.

The complexity of the partial information is:

$$
Q_{\text {partial }}=L \log (F)+Q_{A},
$$

where $Q_{A}$ is the number of bits needed to store the aggregated information $A \cdot \log (F)$ is the total number of bits needed to characterize local states at one subnet. $Q_{A}$ is generally small, and changes slowly with time. Then the complexity of partial information is in the order of $\log F$. We can see that the complexity of the partial information is greatly reduced compared to that of the complete management information especially when the number of wavelengths is large.

A desirable performance-complexity trade-off corresponds to a negligibly small probability of error when only the partial information is used.

\section{Optimal performance using Bayes Rule}

\section{A. Bayes Error}

With partial management information, assessment schemes based on Bayes decision rule [23] achieve the best performance. Given a set of local states $X=\left(N_{1}, N_{2}, \ldots, N_{L}\right)$, the Bayes rule is to decide

$\left\{\begin{array}{l}\omega=1 \quad \text { if } P(\omega=1 \mid X=x)>P(\omega=0 \mid X=x) \\ \omega=0 \text { otherwise, }\end{array}\right.$

where $P(\omega=1 \mid X=x)(P(\omega=0 \mid X=x))$ is a posteriori probability given observation $X=x$. The equality $P(\omega=0 \mid X=x)=P(\omega=1 \mid X=x)$ corresponds to the decision boundary, which divides the space $(X)$ into two regions, $D$ for deciding $\omega=1$ and $\bar{D}$ for deciding $\omega=0$. The Bayes error is the average probability of error as given in Equation (1).

\section{B. Centralized Light-path Assessment}

Such a Bayes rule essentially corresponds to an optimal centralized assessment scheme. Imagine a fictitious central manager, collecting partial information from all subnets. The aggregated information could be polled from each subnet by the central manager at a relatively larger time-scale than the flow dynamics. The local observation $X$ could be collected by the central manager at a smaller time scale. The central manager would then perform the Bayes rule to assess wavelength availability.

This centralized scheme is only conceptual, and used in this work for analysis rather than a practical solution. Centralized assessment may not be feasible for large optical networks because each subnet could belong to different administrative entities. Thus a distributed light-path assessment scheme may be a necessity. However, distributed assessment schemes result in further information loss due to decentralization. Therefore there is a need to understand the best performance achievable using the partial information. Such a best performance would then serve as a basis for assessing the performance of sub-optimal yet practical schemes.

\section{Bayes Error and Blocking Probability}

Although the Bayes error characterizes the optimal performance, it is difficult to evaluate because the decision regions and the corresponding probabilities are hard to obtain. 
Therefore, we derive an upper bound for the Bayes error. Our intent is to relate such a bound with a commonly used network measure such as blocking probability. Such a relation may provide intuition on how error decisions are related to the load $(\rho)$ and wavelength per link $(F)$ of each subnet. For clarity, we describe the blocking probability as given in [7].

Definition 3. The blocking probability $P_{b}$ is defined as the probability that there does not exist a wavelength continuous path to support an end-to-end connection.

A relation between the Bayes error $P_{e}$ and the blocking probability $P_{b}$ can then be derived.

Theorem 1. $0 \leq P_{e} \leq \min \left\{P_{b},\left(1-P_{b}\right)\right\}$.

The proof of the theorem is given in Appendix $A$. Intuitively, the upper bound $\min \left\{P_{b},\left(1-P_{b}\right)\right\}$ can be understood as follows. Consider the following decision rule: If the blocking probability of the network is $P_{b}>1 / 2$, one can reject all connection requests. Otherwise, if $P_{b}<1 / 2$, one can simply accept all requests. This decision rule will have $P_{e}=\min \left\{P_{b},\left(1-P_{b}\right)\right\}$. Since Bayes rule uses local observation $X$ as the additional information for light-path assessment in an optimal fashion, a better performance should be achieved. That is, the Bayes error should be bounded by $\min \left\{P_{b},\left(1-P_{b}\right)\right\}$. The upper bound shows that the probability of error is small if the blocking probability is close to 1 or 0 .

This theorem suggests an analytically feasible way to estimate the Bayes error, which is through the blocking probability. In addition, the bound is obtained independent of a specific model of the blocking probability. The analysis can then be conducted using different models.

\section{Probability of Error under IndePendent Model}

\section{A. Independent Model}

We first assume independent wavelength usage within a subnet and among wavelengths. Such an assumption is equivalent to that all connections within the network are local calls with link-length 1 as shown in Figure 4. Then the corresponding aggregated information is $A=(\rho, F, H, L)$, where $\rho$ is the probability that wavelength is used on one link. The local observation is $X=\left(N_{1}, N_{2}, \ldots, N_{L}\right)$ as defined in Section III. Due to the independent assumption, all the Ni's are independent random variables.

\section{B. Bayes Error}

Under the independent model, the a posteriori probability is

$$
f(X)=P(\omega=1 \mid X)
$$

$$
=\prod_{k=1}^{L}\left(1-\left(1-(1-\rho)^{H-1}\right)^{\left(F-N_{i}\right)}\right),
$$

where $i=1,2, \ldots, L$. This expression means that if $N_{i}$ wavelengths are used at the first hop of subnet $i$, one only needs to decide whether there is one wavelength continuity path at the next $H-1$ hops from $F-N_{i}$ candidate wavelengths. Then $1-\left(1-(1-\rho)^{H-1}\right)^{\left(F-N_{i}\right)}$ is the probability that there is a continuous wavelength at the $i$ th subnet given $N_{i}$, and the product is the probability that the connection request for an end-to-end call can be supported. The Bayes error is:

$$
P_{e}=P(f(X) \geq 1 / 2, \omega=0)+P(f(X)<1 / 2, \omega=1) \text {. }
$$

Equation (5) does not have a close form; and we turn to evaluating the upper bound of $P_{e}$ using the blocking probability of the independent model.

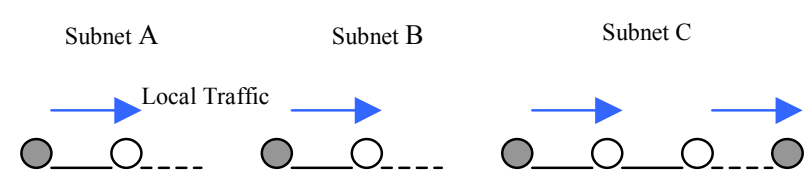

Figure 4: Local calls in independent model

\section{Numerical Analysis}

Under the independent assumption, the probability that there is one end-to-end wavelength continuous path can be obtained using a model in [7]:

$$
P_{a i}=\left(1-\left(1-(1-\rho)^{H}\right)^{F}\right)^{L},
$$

where the sub-index ai means acceptation based on independent model. Therefore, the blocking probability for an end-to-end call is,

$$
P_{b i}=1-\left(1-\left(1-(1-\rho)^{H}\right)^{F}\right)^{L} .
$$

Figure 5 plots the blocking probability $P_{b i}$, (given in Equation (7)) vs. the load ( $\rho$ ) for $F=10,40,120, H=5, L=3$. An interesting phenomenon is that there is a threshold phenomenon on $P_{b i}$. When $\rho$ is below the threshold value (e.g. about at $\rho=0.5$ for $F=120), P_{b i}$ remains close to 0 . When $\rho$ is above the threshold value, $P_{b i}$ increases to 1 rapidly. With a larger $F$, the value of the threshold increases, and the transition of $P_{b i}$ from 0 to 1 gets sharper.

This shows that under most load conditions, we either have a small or a large blocking probability, both of which result in a small probability of error. Therefore, based on Theorem 1, we can conclude that under most load conditions the probability of error for light-path assessment using partial information is small under independent model. Figure 6 confirms this by plotting the upper bound of $P_{e}$ for $F=10,40$, $120, H=5, L=3$. We can see that when the load is close to the threshold, the value of $P_{e}$ increases to the maximum value exponentially; and otherwise, $P_{e}$ is small. 
$P_{b i}$

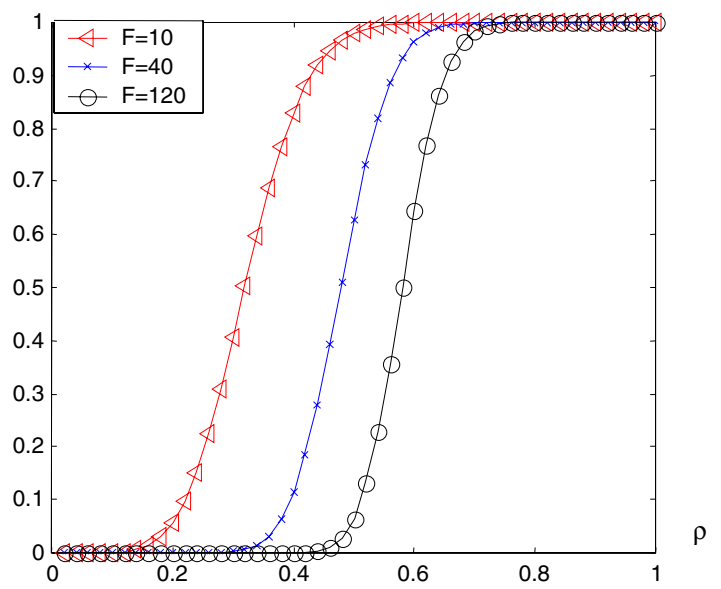

Figure 5. Load $(\rho)$ vs. blocking probability $\left(P_{b i}\right)$ for $\mathrm{F}=10,40,120, \mathrm{H}=5, \mathrm{~L}=3$.

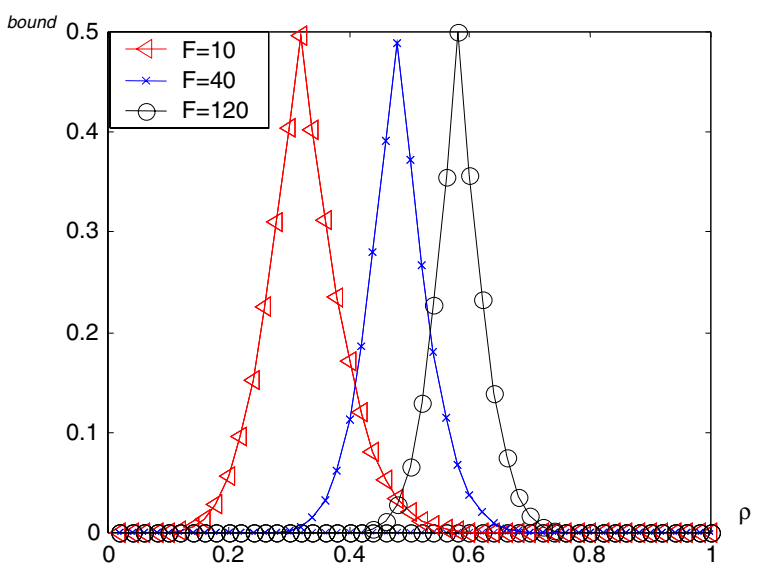

Figure 6 load ( $\rho$ ) vs. the upper bound of $P_{e}$ for $\mathrm{F}=10,40,120, \mathrm{H}=5, \mathrm{~L}=3$.

\section{Special cases}

To quantify the decay rate of the upper bound for large $F$, we consider special cases of low and high load, which correspond to two parts of $P_{b}$ below and above the threshold.

Through algebraic manipulations, we can find that:

(i) When the load is light, i.e., $F>>\frac{1}{(1-\rho)^{H}}$,

$$
0 \leq P_{e} \leq 2 L\left[1-(1-\rho)^{H}\right]^{F} .
$$

(ii) When the load is heavy, i.e., $F \leq \frac{1}{(1-\rho)^{H}}$,

$$
0 \leq P_{e} \leq 2 L(1-\rho)^{F H} \text {. }
$$

These results suggest that the performance trade-off is a small probability of error that decreases exponentially with respect to the number of wavelengths per link $(F)$ under at least moderate and high network load.

\section{Probability of ERror Under Dependent Model}

The above independent model fails to capture the interdomain calls, which extend beyond one subnet. In future optical networks, a significant percentage of the traffic may be transient flows passing through subnets. Therefore, it is important to take the load correlation among subnets into consideration when estimating the performance. In this section, we investigate the probability of error by considering both intra- and inter-domain calls.

\section{A. Dependent Model}

Dependent models in a bus have been investigated in [7] [8] [9]. However, the study in [7] is restricted to having wavelength converters installed at each node, while the network architecture as shown in Figure 2 is with sparselyallocated wavelength converters. More accurate dependent models for the blocking probability on such a topology can be found in [8] [9]. However, both models are complex. Here we extend the dependent model in [7] to obtain a relatively accurate and tractable dependent model for analyzing the probability of error.

To capture the dependence on traffic flows among subnets, we assume that there are two types of calls supported by the network. One corresponds to local calls with hop-length equal to 1 as assumed in the independent model. The other type of call corresponds to inter-domain calls (see Figure 7). Generally, inter-domain calls can originate and/or terminate anywhere at a network. But for simplicity of analysis, we impose the following assumptions:

(i) The inter-domain calls originate and exit only at edge wavelength converters.

(ii) If a wavelength is not used for an inter-domain calls in one subnet, it is used for inter-domain call in the next subnet with probability $P_{n}$.

(iii) If a wavelength is used for one inter-domain call in one subnet, this inter-domain call will exit the current subnet with probability $P_{l,}$, and will continue to the next subnet with probability $1-P_{l}$.

(iv) If a wavelength is used for an inter-domain call in one subnet, and it is released at the edge OXC of this subnet. It is used for inter-domain calls with probability $P_{n}$ in the next subnet.

(v) If an inter-domain call continues to the next subnet, it will use the same wavelength.

(vi) In each subnet, each wavelength is used for a local call in a link with probability $\rho_{1}$, and for and inter-domain call with probability $\rho_{2}$. The probability that a wavelength is used for either a local or an inter-domain call is $\rho=\rho_{1}+\rho_{2}$.

The dependent model captures the link correlation across subnets due to inter-domain calls, and is thus more accurate than the independent model. We are aware that it is limited to assume that the inter-domain calls can only enter or exit at the domain boundaries. However, such a model provides understanding of how inter-domain calls contribute to the 
performance and management information trade-off. A more realistic model is to be investigated in subsequent work.

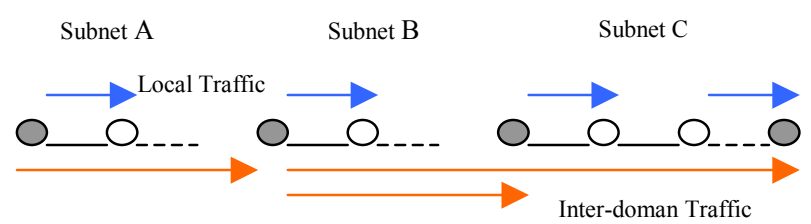

Figure 7: Inter-domain and Local calls

\section{B. Bayes Error}

We begin evaluating the performance by considering the probability of error. Again, we assume that all subnets have identical aggregated information. Under the dependent model, the aggregated information $A$ is $A=\left(\rho_{1}, \rho_{2}, P_{l}, F, H, L\right)$. Local information is the same as that used for independent model, which is $X=\left(N_{1}, N_{2}, \ldots, N_{L}\right)$. Then the a posteriori probability used in Bayes rule is:

$$
\begin{aligned}
f(X) & =P(\omega=1 \mid X) \\
& =\prod_{k=1}^{L}\left(1-\left(1-\left(1-\rho_{c}\right)^{H-1}\right)^{\left(F-N_{i}\right)}\right),
\end{aligned}
$$

where $\rho_{c}=\rho_{1} /\left(1-\rho_{2}\right) \cdot \rho_{c}$ is the probability that a wavelength is used for local calls given that it is not used for inter-domain calls. Such a posterior probability has a similar form to that of the independent case in Equation (4).

The probability of error thus is the same as in Equation (5). But due to inter-domain calls, the local observations (Ni's) at wavelength converters are now dependent random variables. Therefore, the Bayes error is difficult to derive, we turn to study the upper bound based on the blocking probability $P_{b}$.

\section{Blocking Probability}

To derive the blocking probability under the dependent model, we define $\alpha=\rho_{2} / \rho$, which characterizes the percentage of working wavelengths used for inter-domain calls. Then the independent model is just one special case of the dependent with $\alpha=0 \quad\left(\rho_{2}=0\right)$.

From assumptions in Section $A . V I$, we have,

(i) $P_{r}$ (wavelength $w_{j}$ is used for inter-domain call in subnet $i \mid w_{j}$ is not used for inter-domain call in subnet $\left.i-1\right)=P_{n}$

(ii) $P_{r}$ (wavelength $w_{j}$ is used for inter-domain call in subnet $i \mid w_{j}$ is used for inter-domain in subnet $\left.i-1\right)=P_{n} P_{l}+(1$ $\left.P_{l}\right)$.

Therefore,

$$
\rho_{2}=\left(1-\rho_{2}\right) P_{n}+\rho_{2}\left[P_{n} P_{l}+\left(1-P_{l}\right)\right]
$$

It follows

$$
P_{n}=\frac{\rho_{2} P_{l}}{1-\rho_{2}\left(1-P_{l}\right)} .
$$

Define $I_{i}=1$ if there is one wavelength continuous path within subnet $i$; and $I_{i}=0$, otherwise. Then a decision that there are wavelengths available for an end-to-end call $(\omega=1)$ is equivalent to $I_{i}=1$ for all $i$.

Let $M_{i}$ be the number of inter-domain connections in subnet $i$. Then the blocking probability due to the dependent model can be expressed as:

$$
\begin{aligned}
& P_{b d}=1-\sum_{M_{1}, M_{2}, \ldots M_{L}}\left\{P\left(I_{1}=1, I_{2}=1, \ldots, I_{L}=1 \mid M_{1,} M_{2,}, \ldots, M_{L}\right)\right. \\
& =1-\sum_{M_{1}, M_{2}, \ldots M_{L}}\left\{P\left(M_{1}, M_{2}, \ldots, M_{L}\right)\right\} \\
& \left.\ldots P\left(I_{L}=1 \mid M_{L}\right) P\left(M_{1}\right) P\left(I_{2}=1 \mid M_{2}\right) P\left(M_{2} \mid M_{L-1}\right)\right\},
\end{aligned}
$$

where

$$
P\left(I_{i}=1 \mid M_{i}\right)=1-\left[1-\left(1-\rho_{c}\right)^{H}\right]^{\left(F-M_{i}\right)} .
$$

Let $M_{1 i}$ be the number of inter-domain calls in the $i$-th subnet that continue to the next subnet. Since only the $M_{1 i}$ cause the load dependence between the two subnets, we have

$$
P\left(M_{i} \mid M_{i-1}\right)=\sum_{M_{1 i-1}=0}^{M_{i-1}} P\left(M_{i} \mid M_{1 i-1}\right) P\left(M_{1 i-1} \mid M_{i-1}\right),
$$

where

$$
\begin{gathered}
P\left(M_{1 i-1}=m \mid M_{i-1}=k\right)=\left(\begin{array}{l}
k \\
m
\end{array}\right) P_{l}^{k}\left(1-P_{l}\right)^{k-m} \\
\text { for } 0 \leq m \leq k \leq F, \text { and } \\
P\left(M_{i}=h \mid M_{1 i-1}=m\right)=\left(\begin{array}{l}
F-m \\
h-m
\end{array}\right) P_{n}{ }^{(h-m)}\left(1-P_{n}\right)^{F-h} \\
\text { for } 0 \leq m \leq h \leq F .
\end{gathered}
$$

Inserting Equations (14), (15) into Equation (13), $P_{b d}$ can be computed.

\section{Numerical Analysis}

The blocking probability does not have a close-form expression either, but can be evaluated numerically.

Figure 8 plots $P_{b}$ vs. $\rho$ for $F=120, H=5, L=3, \alpha=0,0.6$, $0.9, P_{l}=0.2$. The figure shows that $\rho$ has a similar "threshold effect" on the value of $P_{b}$ to that in the independent model. In addition, the threshold is increasing with $\alpha$, which is defined as the percentage of working wavelengths used for interdomain calls. This, intuitively, is due to the fact that the dependence of wavelength usage introduced by inter-domain calls reduces the blocking probability for a given load $\rho$.

When $\alpha=0$, the dependent model is reduced to the independent model, and the threshold has the lowest value.

Figure 9 plots $P_{b}$ vs. $\rho$ for $F=20,40,120, H=5, L=3$, $\alpha=0.6, P_{l}=0.2$. The figure shows that the threshold is increasing with the number of wavelengths $F$. This is due to the fact that the more wavelengths, the smaller the blocking probability for a given load. Meanwhile, $P_{b}$ seems to experience such a transition for all $F$ consistently. The sharpness of the transition also increases with respect to $F$, 
suggesting an asymptotic behavior of the blocking probability for a large $F$.

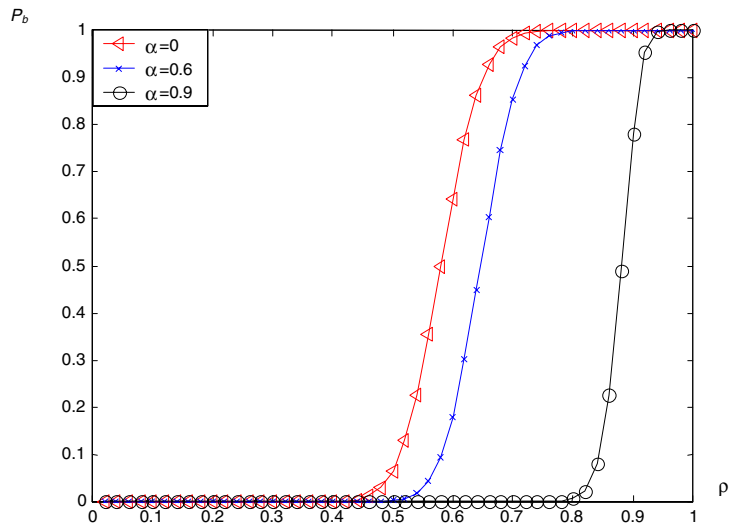

Figure 8 . The load ( $\rho$ ) vs. blocking probability $\left(P_{b}\right)$ for $F=120, H=5, \mathrm{~L}=3, \alpha=0,0.6,0.9, P_{l}=0.2$

$P_{b}$

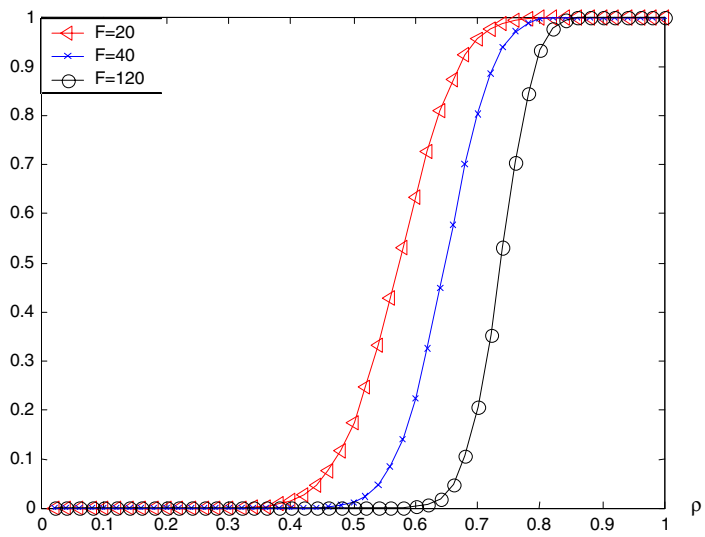

Figure 9 . The load $(\rho)$ vs. blocking probability $\left(P_{b}\right)$ for $F=20,40,120, H=5, L=3, \alpha=0.6, P_{l}=0.2$

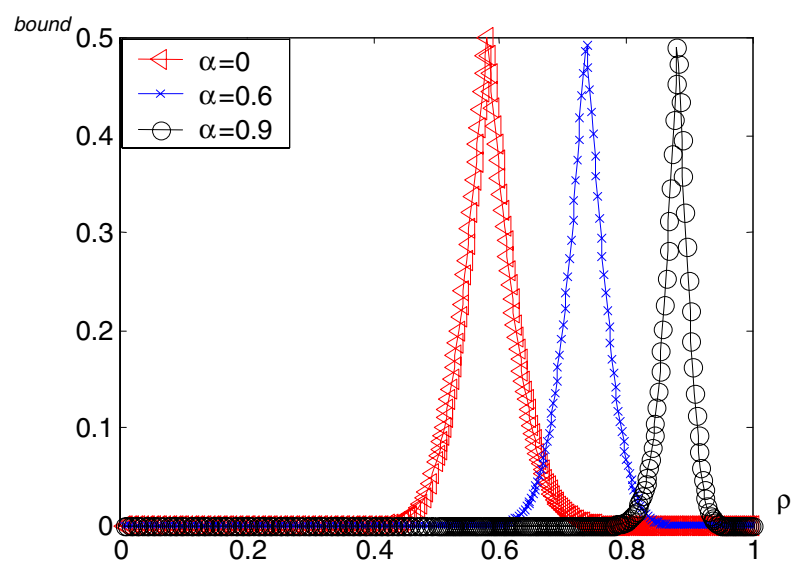

Figure 10. The load $(\rho)$ vs. the uppder bound of $P_{e}$ for $F=120, H=5, L=3, \alpha=0,0.6,0.9, \mathrm{P}_{\mathrm{l}}=0.2$
Figure 10 plots the upper bound for the probability of error from Figure 6 using Theorem 1. The figure shows that the $P_{e}$ is small under most load conditions.

\section{E. Special Cases}

A question rises why the threshold effect persists for both independent and dependent models. We investigate this question by considering special cases when the number of wavelengths is large, and all the sub-networks are weaklyconnected $\left(P_{l}\right.$ is large). Under these conditions, analytical form of the blocking probability can be derived.

\section{E.1 Gaussian Approximation}

An important step to obtain a close form expression for the blocking probability is to approximate the joint probability of the local states $\left(N_{i}\right.$ 's) at wavelength converters. When the number of wavelengths $F$ is large (and $L$ is small), local states at wavelength converters, $X=\left(N_{1}, N_{2}, \ldots, N_{L}\right)$, are joint Gaussian random variables with probability $1-O\left(\frac{L}{\sqrt{F}}\right)$ [24]. Such a Guassian distribution can be completely characterized by the means, variances, and covariances of $N i$ 's. Specifically, all $N_{i}$ 's are random variables with the same mean $\mu$ and variance $\sigma^{2}$, where

$$
\mu=F \rho,
$$

and

$$
\sigma^{2}=F \rho(1-\rho) .
$$

The covariance $C_{i j}$ between $N_{i}$ and $N_{j}$ for $i \neq j$ characterizes the dependence between two subnets, where

$$
C_{i j}=E\left[N_{i} N_{j}\right]-\mu^{2} .
$$

Such dependence can be further characterized through partitioning $N_{i}$ and $N_{j}$ into different components,

$$
\begin{aligned}
& N_{i}=N_{i i}+M_{i t}+M_{i j} ; \\
& N_{j}=N_{j j}+M_{j o}+M_{i j} ;
\end{aligned}
$$

where

$N_{i i}$ is the number of wavelengths occupied by local calls at the first hop of the $i$-th subnet.

$N_{j j}$ is the number of wavelengths occupied by local calls at the first hop of the $j$-th subnet.

$M_{i t}$ is the number of wavelengths in the $i$-th subnet occupied by inter-domain calls that terminate before entering the $j$-th subnet.

$M_{j o}$ is the number of wavelengths in the $j$-th subnet occupied by inter-domain calls that originate after the $i$-th subnet.

$M_{i j}$ is the number of wavelengths used in the $i$-th subnet occupied by inter-domain calls that extend from the $i$-th subnet to the $j$-th subnet.

It is the common factor $M_{i j}$ in $N_{i}$ and $N_{j}$ that causes the dependence between two subnets. Therefore, it is easy to derive that,

$$
C_{i j}=\operatorname{var}\left[M_{i j}\right]
$$


Furthermore, the average number of inter-domain calls is $F \rho_{2}$. The probability for an inter-domain call in subnet $i$ to extend to subnet $j$ is

$$
\begin{aligned}
P_{i j} & =1-P_{l} \sum_{k=0}^{j-i-1}\left(1-P_{l}\right)^{k} \\
& =\left(1-P_{l}\right)^{j-i} .
\end{aligned}
$$

Then due to the binomial nature of $M_{i j}$,

$$
C_{i j}=F \rho_{2} P_{i j}\left(1-P_{i j}\right) \text {, }
$$

and the correlation coefficient is $\frac{C_{i j}}{\sigma_{N_{i}}^{2}}$. In particular,

$$
\rho_{i j}=\frac{\rho_{2} P_{i j}\left(1-P_{i j}\right)}{\rho(1-\rho)} .
$$

\section{E.2 Weakly-Connected Sub-Networks}

When $\rho_{i j}=0$, all sub-networks are completely decoupled, i.e., each inter-domain call lasts for one subnet $\left(P_{l}=1\right)$. Specifically, for the non-blocking probability of decoupled subnets, we have

$$
P_{a d}^{*}=\left\{1-\left[1-(1-\rho)\left(1-\rho_{c}\right)^{H-1}\right]^{F}\right\}^{L},
$$

where $\rho_{c}$ is the probability that a wavelength is used for local calls given that it is not used for inter-domain calls. In particular,

$$
\rho_{c}=\rho_{1} /\left(1-\rho_{2}\right) .
$$

For the non-blocking probability of the independent model, we have

$$
P_{a i}=\left\{1-\left[1-(1-\rho)^{H}\right]^{F}\right\}^{L} .
$$

Clearly, Equation (26) bears a similar form to Equation (6), and thus simple algebraic manipulations show that there exists a threshold effect in the blocking probability for decoupled subnets similar to that for the independent model.

When $\rho_{i j}$ 's are small, all sub-networks are weaklyconnected, i.e., a small percentages of the calls are interdomain calls ( $\alpha$ is small), and/or inter-domain calls exit at current subnet with a large probability $\left(P_{l}\right.$ is large). Through Taylor Expansion, we have the following theorem for weakly connected sub-networks.

Theorem 2. For weakly-connected sub-networks, i.e., $\alpha$ is small and/or $P_{l}$ is large, the non-blocking probability of the dependent model can be expressed as

$$
P_{a d}=P_{a d}^{*}(1+\eta)+o\left(\max \left\{\rho_{i j}\right\}\right),
$$

where $P_{a d}^{*}$ is the non-blocking probability of the decoupled subnets as in equation (26).

Detailed derivations of $\eta$, and the proof of the theorem can be found in Appendix B.

The weakly-connected subnets exhibit interesting properties under the following conditions: (i) When $P_{l}=1$, all inter-domain calls last one subnet. Hence all the sub-networks are decoupled, and we have $\eta=0$, $P_{a d}=P_{a d}^{*}$.

(ii) When $P_{l}$ is large (e.g. $P_{l} \geq 0.9$ ), a small percentage of the inter-domain calls last more than one subnets. Hence the sub-networks are weakly-connected, and $P_{a d} \approx P_{a d}^{*}(1+\eta)$. Here the non-blocking probability is just that of the decoupled sub-networks plus a small perturbation. Thus we can expect a threshold effect occurs under the weakly-connected subnetworks.

(iii) When $P_{l}=0$, all the inter-domain calls are end-toend. The blocking probability essentially reduces to that for (independent) local calls when a certain number of wavelengths is occupied by inter-domain calls. Then $\rho_{i j}=0, P_{a d}=P_{a d}^{*}$. There is also a threshold effect for the blocking probability as shown in Section $V$.

The analysis here further explains why the threshold effect persists for both independent and dependent model when $F$ is large for some special cases.

\section{Conclusion}

In this paper, we investigate network management information for light-path assessment across administrative domains (subnets). Our focus has been on studying the best performance achievable in assessing wavelength availability using partial management information. The partial management information includes aggregated information of subnets, and local states from wavelength converters on the network-domain boundaries. The complexity of partial information is thus much reduced compared with the detailed and complete state information.

We have formulated the problem based on decision theory, and defined the performance of using partial management information through the Bayes probability of erroneous assessment. A bound in terms of the blocking probability is derived to estimate such a performance. We have evaluated the blocking probability and the upper bound for both independent and dependent models of wavelength usage. Our study reveals an interesting phenomenon that, when the number of wavelengths is large, the blocking probability transits from 0 to 1 rapidly. This results in a Bayes error negligibly small for most of the network load conditions. Such results suggest that a small loss in performance (the Bayes error) may result in a large saving in network management information. That is, the abundant network resource, which is the large number of wavelengths of future WDM networks may make it possible to reduce the amount of network management information while achieving a good performance.

Our formulation and analysis are based on simple models for intra- and inter-domain calls. Other models will be examined in the future. In this work, we studied the performance-complexity tradeoff of a special case of partial 
information. Other forms of management information will be investigated also. Another future direction is to extend this work to a more general network topology, and to implement good light-path assessment schemes.

\section{ACKNOWLEDGMENT}

CJ gratefully acknowledges hospitality from LIDS, MIT where this work was initiated during her visit. The authors would like to thank Eytan Modiano for sharing with us his early work on wavelength routing, Muriel Medard for helpful discussions, and Zesheng Chen for his help with the improvement of the bound. Support from National Science Foundation (ECE9908578) and Yamacraw Center at Georgia Tech is gratefully acknowledged.

\section{APPENDIX A \\ PROOF OF THEOREM 1}

Proof:

Consider the following a posterior probability:

$$
f(x)=P(\omega=1 \mid X=x) .
$$

The Bayes rule decides

$$
\left\{\begin{array}{l}
\omega=1 \quad \text { if } f(x) \geq 1 / 2 \\
\omega=1 \quad \text { otherwise }
\end{array}\right.
$$

Therefore,

$$
\begin{aligned}
& P_{e}=\sum_{X} P_{e \mid X=x} P(X=x) \\
& \quad=\sum_{X} P(X=x) \min \{f(x),(1-f(x))\} \\
& \quad \leq \min \left\{\sum_{X} P(X=x) f(x), \sum_{X} P(X=x)(1-f(x))\right\} . \\
& \text { Since } P_{b}=1-\sum_{X} P(X=x) f(x),
\end{aligned}
$$

we have

$$
\begin{gathered}
0 \leq P_{e} \leq \min \left\{P_{b},\left(1-P_{b}\right)\right\} . \\
\text { APPENDIX B } \\
\text { PROOF OF THEOREM } 2
\end{gathered}
$$

Proof:

The non-blocking probability of the dependent model satisfies

$$
P_{a d}=E[f(\omega=1 \mid X)] .
$$

Since $X=\left(N_{1}, \ldots, N_{L}\right)$ is joint Gaussian, we can expand $P_{a}$ in terms of $\rho_{i j}$ 's as follows,

$$
P_{a d}=P_{a d}^{*}+\sum_{i \neq j} K_{i j} \rho_{i j}+o\left(\max \left\{\rho_{i j}\right\}\right),
$$

where $P_{a d}^{*}$ is the non-blocking probability of the dependent model when all the inter-domain calls only last for one subnet $\left(P_{l}=1\right)$. Specifically, we have

$$
\begin{gathered}
P_{a d}^{*}=\left\{1-\left[1-(1-\rho)\left(1-\rho_{c}\right)^{H-1}\right]^{F}\right\}^{L}, \\
K_{i j}=\left.\frac{\partial P_{a}}{\partial \rho_{i j}}\right|_{\rho_{i j}=0, \forall i \neq j},
\end{gathered}
$$

which is soon shown to be the same for all $i, j$ 's.

$$
K_{i j}=\left\{1-\left[1-(1-\rho)\left(1-\rho_{c}\right)^{H-1}\right]^{F}\right\}^{L-2} q_{i j},
$$

where

$q_{i j}=\left.\iint_{-\infty}^{\infty}\left(1-\gamma^{F-N_{i}}\right)\left(1-\gamma^{F-N_{j}}\right) \frac{\partial f\left(\pi_{i}, \pi_{j} ; \rho_{i j}\right)}{\partial \rho_{i j}}\right|_{\rho_{i, j=0}} d N_{i} d N_{j}$

with $f\left(\pi_{i,} \pi_{j ;} \rho_{i j}\right)$ being the joint Gaussian p.d.f. of $N_{i}$ and $N_{j}$, and $\gamma=\left[1-\left(1-\rho_{c}\right)^{H-1}\right]$.

Simplifying $q_{i j}$, we have,

$$
\begin{gathered}
q_{i j}=\frac{1}{F \rho(1-\rho)}\left\{\iint_{-\infty}^{\infty} f\left(\pi_{i}, \pi_{j}, 0\right)\left(N_{i}-F \rho\right)\left(N_{j}-F \rho\right)\right. \\
\left.\left(1-\gamma^{F-N_{i}}\right)\left(1-\gamma^{F-N_{j}}\right) d N_{i} d N_{j}\right\} .
\end{gathered}
$$

Let $\mu=F \rho$ and $\sigma^{2}=F \rho(1-\rho)$, we have

$$
q_{i j}=\gamma^{2(F-\mu)+\sigma^{2} \ln \gamma} \sigma^{2} \ln ^{2} \gamma,
$$

which is not dependent on the value of $i$ and $j$.

$$
\begin{array}{r}
\text { Since } \rho_{i j}=\frac{\rho_{2} P_{i j}\left(1-P_{i j}\right)}{\rho(1-\rho)} \text { and } P_{i j}=\left(1-P_{l}\right)^{j-i}, \\
\rho_{i j}=\frac{\rho_{2}\left[\left(1-P_{l}\right)^{(j-i)}-\left(1-P_{l}\right)^{2(j-i)}\right]}{\rho(1-\rho)} .
\end{array}
$$

Then

$$
\begin{aligned}
& \sum_{1 \leq i \leq j \leq L} \rho_{i j}=\frac{\rho_{2}\left[(L-1) \rho_{12}+(L-2) \rho_{13}+\ldots+\rho_{1 L}\right]}{\rho(1-\rho)} \\
& =\left[\frac{(L-1)\left(1-P_{l}\right)}{P_{l}\left(2-P_{l}\right)}+\frac{\left(1-P_{l}\right)^{2}\left(3-2 P_{l}^{2}\right)}{P_{l}^{2}\left(2-P_{l}\right)^{2}}+o\left(\frac{\left(1-P_{l}\right)^{L+1}}{P_{l}^{2}}\right)\right] \\
& \frac{\rho_{2}}{\rho(1-\rho)} .
\end{aligned}
$$

Therefore, we have

$$
\begin{gathered}
P_{a d}=P_{a d}^{*}(1+\eta)+o\left(\max \left\{\rho_{i j}\right\}\right), \\
\text { Where } \eta=\frac{\left\{1-\left[1-\left(1-\rho_{c}\right)^{H-1}\right]^{F}\right\}^{2}}{\left\{1-\left[1-(1-\rho)\left(1-\rho_{c}\right)^{H-1}\right]^{F}\right\}^{2}} q_{i j} \sum_{1 \leq i \leq j \leq L} \rho_{i j} .
\end{gathered}
$$

\section{REFERENCES}

[1] Admela Jukan and Harmen R. van As, "Service-Specific Resource Allocation in WDM Networks with Quality Constraints," IEEE Journal on Selected Areas in Communications, VOL. 18, NO. 10, October 2000.

[2] Paul Green, "Progress in Optical Networking," IEEE Communications Magazine, January 2001.

[3] R. Ramaswarmi, K. Sivarajan, Optical Networks, A practical Perspecitve, The Morgan Kaufman Publishers, San Fransico, 1998.

[4] Yong Xue, Monica Lazer, Ananth Nagarajan, Olga Aparicio and Steven Wright, "Carrier Optical Services Requirements," IETF Draft, March 2002.

[5] Personal Communication with D. Mitra. 
[6] R. Guerin and A. Orda, "QoS Routing in Networks with Inaccurate Information: Theory and Algorithms," IEEE/ACM Trans. Networking, Vol. 7, No. 3, June 1999.

[7] Richard A. Barry and Pierre A. Humblet, "Models of Blocking Probability in All-Optical Networks with and Without Wavelength Changers," IEEE Journal on Selected Areas in Communications, VOL. 14, NO. 5, June 1996.

[8] Yuhoung Zhu, George N. Rouskas and Harry G. Perros, "A Path Decomposition Approach for Computing Blcoking Probabilities in Wavelength Routing Networks," IEEE/ACM Transactions on Networking, Vol. 8, No. 6, December 2000.

[9] S. Subramaniam, M. Azizoglu, and A. K. Somani, "All-optical networks with sparse wavelength conversion," IEEE/ACM Transactions on Networking, vol. 4, no. 4, pp. 544-57, Aug. 1996.

[10] R. Ramaswami, A. Segall, "Distributed Network Control for Wavelength Routed Optical Network," IEEE/ACM Transactions on Networking, Vol. 5, No. 6, December 1997.

[11] Daniel Awduche, Yakov Rekhter, "Multiprotocol Lambda Switching: Combining MPLS Traffic Engineering Control with Optical Crossconnects," IEEE Communications Magazine, March 2001.

[12] N. Chandhok, A. Durresi, R. Jagannathan, R. Jain and K. Vinodkrishnan, "IP over Optical Networks: A Summary of Issues," IETF Draft, March 2001.

[13] Murali Kodialam, T. V. Lakshman, "Dynamic routing of locally restorable bandwidth guaranteed tunnels using aggregated link usage information," INFOCOM 2001, April 2001.

[14] Chunming Qiao and Dahai Xu, "Distributed Partial Information Management (DPIM) Schemes for Survivable Networks - Part I," Infocom 2002, June, 2002

[15] Hungjen Wang, Eytan Modiano and Muriel Medard, "Partial Path Protection for WDM Networks: End-to-End Recovery Using Local Failure Information," IEEE JSCC, July 2002.

[16] Ling Li and Arun K. Somani, "Dynamic Wavelength Routing Using Congestion and Neighborhood Information," IEEE/ACM Trans. On Networking, Volume 7, No. 5, October 1999.

[17] A. Elwalid, C. Jin, S. Low, I. Widjaja, "MATE: MPLS Adaptive Traffic Engineering," Proc. Infocom 2001, April 2000.

[18] J. Yates, "Wavelength Converters in Dynamically-Reconfigurable WDM Nerworks," IEEE Communications Surveys, Second Quarter 1999.

[19] S. Subramaniam, M. Azizoglu, and A.K. Somani, " On Optimal Converter Placement in Wavelength-Routed Networks," IEEE/ACM Trans. Networking, VOL. 7, No.5, October 1999.

[20] K.S. Lui, K. Nahrstedt, "Topology Aggregration and Routing in Bandwidth-Delay Sensitive Network," Proc. of IEEE Globecom 2000, San Fransicisco, CA, November-December, 2000.

[21] S. Nelakuditi, Z.L. Zhang, and R. P. Tsang "Adaptive Proportional Routing: A Localized QoS Routing Approach," Proc. IEEE INFOCOM'00, Tel Aviv, Israel, March 2000.

[22] G. Liu, C. Ji , and V. Chan "Network Management Information for Light-paht Assessment," ISIT 2002, Lausanne, Switzerland, June 2002.

[23] Richard O. Duda and Peter E. Hart, Pattern Classification, Wiley, New York, 2001

[24] William Feller, An Introduction to Probability Theory and Its Applications, $3^{\text {rd }}$ edition, Vol. 1, John Wiley \& SONS, INC. New York, N.Y. 